\title{
Effects of type 1 diabetes mellitus on cardiac function: a study of monozygotic twins
}

\author{
S S S Lo, R D G Leslie, M St John Sutton
}

Department of Diabetes and Metabolism, St Bartholomew's Hospital, London S S S Lo

R D G Leslie

Royal Brompton

Hospital, London

M St John Sutton

Correspondence to:

Dr Simon S S Lo,

Department of Cardiology,

Royal Brompton National

Heart and Lung Hospital

Sydney Street, London,

SW3 6NP.

Accepted for publication

5 December 1994

\begin{abstract}
Objective-To investigate left ventricular size and function in type 1 diabetes and their relation with diabetes duration, glycaemic control, autonomic dysfunction, and complications of diabetes.

Design-Cross sectional study using a pulsed wave Doppler echocardiogram to assess left ventricular dimensions, wall thickness, and transmitral blood flow velocity signals.

Patients-40 monozygotic twin pairs (23 male, mean age 26 years) discordant for type 1 diabetes and 40 non-diabetic singleton controls with no clinical evidence of cardiac ischaemia.

Results-For all Doppler echocardiographic measurements there were strong correlations between monozygotic twins but not between twins and control subjects. Left ventricular dimensions, wall thickness and systolic function, peak $E$ velocity, and the velocity integrals of early left ventricular filling were similar in all three groups. Peak $A$ velocity and the velocity integrals of late ventricular filling (mean (SD)) were greater in diabetic twins $(45$ (12) $v 38(8) \mathrm{cm} / \mathrm{s}, P=$ 0.002 ; and 32 (11) $v 26(6), P=0.0002)$. Diabetic twins had lower E/A ratio (1.59 $(0.39) v 1.83(0.39), P<0.001)$, greater atrial filling fraction to total diastolic filling (28 (6) $v 25(5) \%, P=0.002)$, and prolonged isovolumic relaxation time $(72$ (12) $v 63$ (9) $\mathrm{ms}, P<0.001$ ). The differences in Doppler findings between diabetic and non-diabetic twins were related to disease duration whereas the prolongation of the isovolumic relaxation time was related to cardiac autonomic dysfunction.

Conclusions-These results show that twins with type 1 diabetes have left ventricular diastolic dysfunction related to diabetes duration and cardiac autonomic dysfunction but not to glycaemic control or microvascular complications. In addition, genetic factors contribute to left ventricular dimension and function.
\end{abstract}

(Br Heart f 1995;73:450-455)

Keywords: diabetes; twins; ventricular function

Diabetic patients develop heart failure more frequently than non-diabetic patients, despite a similar extent of coronary artery disease. ${ }^{1}$ The
Framingham study suggested that a "specific diabetic cardiomyopathy" unrelated to coronary atherosclerosis could account for this excess heart failure. ${ }^{2}$ The characteristics of this putative diabetic cardiomyopathy have never been clearly defined. Echocardiographic studies have shown normal and abnormal left ventricular dimensions and systolic function in diabetic patients, ${ }^{3-5}$ while diastolic function was often impaired. ${ }^{5-7}$ However, the relations between myocardial dysfunction and the type and duration of diabetes, the impact of glycaemic control, microvascular disease, and abnormal autonomic function remain unclear. We studied monozygotic twins discordant for type 1 diabetes to examine the direct effects of type 1 diabetes on left ventricular size and function, and to determine whether there is evidence for a specific diabetic heart muscle disease, and if so whether it is related to disease duration, glycaemic control, the presence of microvascular disease, or autonomic dysfunction. The non-diabetic co-twins are the ideal controls because the well documented influence of genetic factors on left ventricular mass and size were eliminated and the environmental effects minimised by their shared family backgrounds.

\section{Patients and methods}

STUDY POPULATION

The British Diabetic Twin Study has 101 pairs of monozygotic twins discordant for type 1 diabetes. We selected all 47 twin pairs between the ages of 10 and 37 years for this study because diabetic patients of this age have been shown to have a low incidence of coronary artery disease ${ }^{589}$ and we wanted to exclude the confounding effects of coexistent coronary heart disease on left ventricular function. We compared these twins with a group of age and sex matched volunteers who were recruited from the Borough of Westminster.

Type 1 diabetes was diagnosed according to the National Diabetes Data Group criteria $^{10}$ and non-diabetic twins were tested with an oral glucose tolerance test to exclude diabetes. ${ }^{10}$ Zygosity was defined by 21 genetic markers and the probability of monozygosity was greater than 95\%." Exclusion criteria were $(a)$ history of cardiac ischaemia; $(b)$ hypertension (blood pressure $>160 / 95 \mathrm{~mm} \mathrm{Hg}$ ); $(c)$ valvar heart disease or segmental wall motion abnormalities on the cross sectional echocardiogram; (d) abnormal 12 lead electrocardiogram, especially atrial fibrillation or 
bundle branch block; (e) alcohol intake of $>35$ units per week; $(f)$ renal dysfunction (serum creatinine $>150 \mu \mathrm{mol} / \mathrm{l})$; $(g)$ diabetic ketoacidosis two months before study; and $(h)$ medications known to affect cardiac function. Seven of the 47 twin pairs, were excluded: three with hypertension; one with anorexia nervosa; one was taking growth hormone therapy and two pairs did not wish to participate in the study. Thus the study consisted of 40 twin pairs. It was approved by Riverside Hospital ethics committee.

Subjects were requested to grade their weekly exercise activities: grade $1=$ sedentary (office work, irregular non-strenuous exercise); grade 2 = fit (regular exercise up to three times per week-for example, brisk walking, games and sports), and grade $3=$ athletic (regular weight training, track running, and cycling). In addition, smoking history and daily insulin dose were recorded. Supine blood pressures were measured after five minutes rest with a cuff sphygmomanometer, and repeated at least twice immediately before echocardiographic recordings. Mean blood pressure (BP) was calculated as (systolic $\mathrm{BP}$ - diastolic $\mathrm{BP}$ )/3 plus diastolic BP.

ASSESSMENT OF CARDIAC SIZE AND FUNCTION Doppler echocardiograms were obtained with simultaneous electrocardiographic lead II using a 2.5 or $3.75 \mathrm{MHz}$ focused transducer (Toshiba Sonolayer SSH-160A). The ultrasonographer (MSS) was blinded to the subjects' diabetes status. $M$ mode echocardiograms of the left ventricle were recorded (with cross sectional echocardiographic guidance) at the tips of the mitral valve leaflets to measure left ventricular dimensions and wall thickness together with a phonocardiogram in order to measure isovolumic relaxation time (IVRT). Pulsed wave Doppler transmitral blood flow velocity signals were recorded from the apical four chamber view with the sample volume positioned in the middle of the flow stream at the tips of the mitral valve leaflets. $M$ mode echocardiograms and Doppler blood flow velocity signals were recorded on a strip-chart recorder at a paper speed of $100 \mathrm{~mm} / \mathrm{s}$.

Table 1 Diabetic complication index

\begin{tabular}{ll}
\hline Complication & Grades \\
\hline Retinopathy: & \\
None 0 & \\
Microaneurysms, haemorrhages, and exudates & 1 \\
Preproliferative and proliferative changes & 2 \\
Micralbuminuria; & 0 \\
$<15 \mu \mathrm{g} / \mathrm{min}$ & 1 \\
$>15 \mu \mathrm{g} / \mathrm{min}$ & \\
Autonomic function tests: & 0 \\
HRB VR All tests normal & 1 \\
HRS Y One or two abnormal tests & 2 \\
PBP Three or four abnormal tests &
\end{tabular}

Abnormal autonomic function tests were defined as $>2 \mathrm{SD}$ below the mean value derived from the healthy controls. HRB, heart rate variation in response to deep breathing; VR, Valsalva ratio; HRS, heart rate increase on standing; PBP, postural hypotension if $>20 \mathrm{~mm} \mathrm{Hg}$ fall in systolic BP. The maximum score for each patient is 5 .
ASSESSMENT OF DIABETIC MICROVASCULAR COMPLICATIONS

Retinopathy was graded 0 to 2 from retinal photographs by a consultant ophthalmologist who had no knowledge of diabetic status (table 1).

Nephropathy-microalbumin excretion rate was measured from a timed overnight urine collection by immunonephelometric method (upper limit of normal $15 \mu \mathrm{g} / \mathrm{min}$ ). A further sample was collected if the initial sample showed microalbuminuria (proteinuria $>200 \mu \mathrm{g} / \mathrm{min}$ ) and mean values were calculated. Microalbuminuria was graded as in table 1 .

\section{ASSESSMENT OF AUTONOMIC NEURAL}

FUNCTION

Autonomic function was assessed using a panel of standard cardiovascular reflex tests with simultaneous electrocardiographic monitoring as previously described ${ }^{12}(a)$ beat to beat heart rate variation to deep breathing; $(b)$ mean ratio of fastest to slowest heart rates during two Valsalva manoeuvres; (c) heart rate increase on standing; (d) blood pressure changes on standing. The results were graded as in table 1.

\section{LABORATORY ASSESSMENTS}

Glycaemic control was assessed by glycated haemoglobin concentration (HbAl) determined by electroendosmosis (Corning method (normal range 3.5-8.5\%); Serum total cholesterol concentrations were measured by an autoanalyser.

\section{ECHOCARDIOGRAPHIC DATA ANALYSIS}

$M$ mode echocardiograms were analysed in a blinded fashion from at least three cardiac cycles as described by the American Society of Echocardiography. ${ }^{13}$ Measurements included (a) left ventricular end diastolic dimension (LVEDD) and end systolic dimension (LVESD); (b) fractional shortening (FS) = (LVEDD - LVESD)/LVEDD $\times 100 \%$; $(c)$ interventricular and posterior wall thickness; (d) IVRT measured as the time period between the opening of the mitral valve leaflets detected by $M$ mode echocardiograms and the first high frequency component of the aortic valve closure sound detected by phonocardiography. All echocardiographic measurements were corrected for body surface area. Transmitral Doppler flow velocity signals from a minimum of five cardiac cycles were digitised to obtain (a) peak velocity during early left ventricular diastolic filling ( $E$ wave); (b) peak velocity during late (atriosystolic) left ventricular diastolic filling (A wave); (c) ratio of peak flow velocities (E/A); (d) flow velocity integrals (FVI $=$ area under the time velocity curve) during early and late diastolic filling; (e) atrial filling fraction, which is the fraction of left ventricular filling contributed by atrial systole, calculated as FVIA/(FVIA + FVIE) $\times$ $100 \%$. 
Table 2 Clinical, metabolic, and haemodynamic data $(n=40)($ mean $(1 S D))$

\begin{tabular}{|c|c|c|c|}
\hline Characteristics & $\begin{array}{l}\text { Diabetic } \\
\text { twins }\end{array}$ & $\begin{array}{l}\text { Non-diabetic } \\
\text { twins }\end{array}$ & Controls \\
\hline Age (yr) & $26(8)$ & $26(8)$ & $26(7)$ \\
\hline $\operatorname{Sex}(M / F)$ & $23 / 17$ & $23 / 17$ & $23 / 17$ \\
\hline Age at diagnosis of diabetes & $13(7)$ & - & - \\
\hline Duration of diabetes (yr) & $14(7)$ & - & - \\
\hline Insulin dose (U/day) & 50 & - & - \\
\hline $\mathrm{BSA}\left(\mathrm{m}^{2}\right)$ & $1 \cdot 71(0 \cdot 2)$ & $1 \cdot 74(0 \cdot 3)$ & $1.75(0.2)$ \\
\hline BMI $\left(\mathrm{kg} / \mathrm{m}^{2}\right)$ & $22 \cdot 8(3 \cdot 2)$ & $23 \cdot 0(3 \cdot 8)$ & $22 \cdot 0(2 \cdot 6)$ \\
\hline Smokers & 7 & 11 & 8 \\
\hline Serum creatinine $(\mu \mathrm{mol} / \mathrm{l})$ & $85(11)$ & $85(12)$ & $89(11)$ \\
\hline Total cholesterol $(\mathrm{mmol} / \mathrm{l})$ & $4 \cdot 8(1 \cdot 2)$ & $4 \cdot 6(0 \cdot 9)$ & $4 \cdot 4(0 \cdot 8)$ \\
\hline $\mathrm{HbAl}(\%)$ & $11 \cdot 1(2 \cdot 6)^{\star \star}$ & $6 \cdot 4(1 \cdot 2)$ & - \\
\hline Heart rate (beats/min) & $80(14)^{\star} \dagger$ & $71(14)$ & $71(10)$ \\
\hline Systolic BP (mm Hg) & $119(10)^{\star \star}$ & $114(8)$ & $117(8)$ \\
\hline Diastolic BP (mm Hg) & $75(8)^{\star \star}$ & $70(8) \ddagger$ & $74(6)$ \\
\hline Mean BP $(\mathrm{mm} \mathrm{Hg})$ & $89(8)^{\star \star}$ & $84(7)$ & $88(8)$ \\
\hline
\end{tabular}

BSA, body surface area; BMI, body mass index. Statistical significant difference between twins ${ }^{\star} \mathrm{P}<0.02$ and $\star \star \mathrm{P}<0.002$; between diabetic twins and control subjects $+\mathrm{P}<0.001$; and between non-diabetic twins and control subjects as $¥ P<0.005$.

Table $3 M$ mode echocardiographic data $(n=40)($ mean $(1 S D))\left(\mathrm{cm} / \mathrm{m}^{2}\right)$

\begin{tabular}{llcc}
\hline Variable & $\begin{array}{l}\text { Diabetic } \\
\text { twins }\end{array}$ & $\begin{array}{l}\text { Non-diabetic } \\
\text { twins }\end{array}$ & \multicolumn{1}{c}{ Controls } \\
\hline LVEDDi & $2 \cdot 80(0 \cdot 27)$ & $2 \cdot 84(0 \cdot 29)$ & $2 \cdot 86(0 \cdot 26)$ \\
LVESDi & $1 \cdot 77(0 \cdot 23)$ & $1 \cdot 81(0 \cdot 22)$ & $1 \cdot 76(0 \cdot 19)$ \\
PwTi & $0.50(0 \cdot 07)$ & $0 \cdot 50(0.06)$ & $0 \cdot 49(0.04)$ \\
IVSTi & $0.51(0.07)$ & $0 \cdot 50(0.07)$ & $0 \cdot 50(0.05)$ \\
FS (\%) & $37(5)$ & $37(4)$ & $38(5)$ \\
\hline
\end{tabular}

All variables except fractional shortening (FS) were indexed (i) to body surface area. LVEDD and LVESD are left ventricular end diastolic and end systolic dimensions respectively; PwT and IVST are posterior wall and interventricular septal thickness respectively. FS, fractional shortening.

Table 4 Doppler echocardiographic data $(n=40)$ and IVRT $(n=33)($ mean $(1 S D))$

\begin{tabular}{llll}
\hline Variable & $\begin{array}{l}\text { Diabetic } \\
\text { twins }\end{array}$ & $\begin{array}{l}\text { Non-diabetic } \\
\text { twins }\end{array}$ & Controls \\
\hline E velocity $(\mathrm{cm} / \mathrm{s})$ & $69(13)$ & $67(12)$ & $71(14)$ \\
A velocity $(\mathrm{cm} / \mathrm{s})$ & $45(12)^{\star \star} \dagger$ & $38(8) \oint$ & $33(6)$ \\
E/A & $1 \cdot 59(0 \cdot 39)^{\star \star} \dagger$ & $1 \cdot 83(0 \cdot 39)$ & $2 \cdot 19(0 \cdot 55)$ \\
FVIE & $80(20)$ & $78(13)$ & $84(17)$ \\
FVIA & $32(11)^{\star \star \dagger}$ & $26(6) \ddagger$ & $23(6)$ \\
Total FVI & $112(27)$ & $104(16)$ & $107(20)$ \\
A filling \% & $28(6)^{\star \star \dagger}$ & $25(5) \oint$ & $21(5)$ \\
IVRT (ms) & $72(12)^{\star \star \dagger}$ & $63(9)$ & $61(13)$ \\
\hline
\end{tabular}

FVIE and FVIA are flow-velocity integrals during early and late ventricular diastolic filling respectively. A filling \% is the percentage of left ventricular filling contributed by left atrial systolic contraction. IVRT is isovolumic relaxation time. Statistical significant difference between twins non-diabetic twins and controls as $¥ P<0.02$ and $\$ P<0.001$. In seven twins, the separation of the non-diabetic twins and controls as $¥ P<0.02$ and $₫ P<0.001$. In seven twins,
mitral valve leaflets were not clearly defined on $M$ mode echocardiogram.

\section{STATISTICAL ANALYSIS}

Results were presented as mean (SD), and $P$ $<0.05$ was regarded as significant. The null hypothesis within twin pairs and between twins and matched control subjects was examined by Student's paired $t$ test. In addition, $95 \%$ confidence intervals (CI) for the differences between the means of the groups were presented in the text where appropriate. Stepwise multiple regression analysis (Apple StatView 512+ software) was used to examine the effects of diabetes duration, blood pressure, heart rate, $\mathrm{HbAl}$ concentrations, and exercise activity grades on echocardiographic measurements of left ventricular dimension and function. The association between the diabetic complication index and echocardiographic measurements were examined by Spearman's rank correlation coefficient. The $\chi^{2}$ test was used to evaluate the frequency of retinopathy, nephropathy, and autonomic dysfunction in the diabetic and non-diabetic twins.

\section{Results}

PATIENT CHARACTERISTICS

The mean age of the twins (23 male, 17 female) and controls (23 male, 17 female) was 26 years (range 10-37) and the mean duration of diabetes was 14 years (table 2). Mean heart rate was significantly higher in the diabetic twins than in the non-diabetic twins and control subjects (table 2). Systolic, diastolic and mean blood pressures were significantly higher in diabetic twins than in non-diabetic twins, but were similar to controls (table 2). The twin pairs performed similar degrees of physical activity, with 26 pairs independently grading themselves in the same category, so that the Spearman rank correlation for exercise activity among the twin pairs was 0.62 .

\section{MODE ECHOCARDIOGRAPHIC DATA}

Left ventricular end diastolic and end systolic dimensions, posterior wall and interventricular septal thickness, and fractional shortening were similar in all three groups (table 3 ).

\section{DOPPLER ECHO AND IVRT}

The mean peak $\mathrm{E}$ velocity amplitude and the $E$ velocity integral were similar in all three groups (table 4). By contrast, the mean peak A velocity amplitude and the A velocity integral were significantly greater in the diabetic twins than their non-diabetic co-twins $(95 \%$ CI 2.15 to $9.4 \mathrm{~cm} / \mathrm{s}, \mathrm{P}<0.01$ ) and controls (95\% CI 6.57 to $14.53 \mathrm{~cm} / \mathrm{s}, \mathrm{P}<0.001)$. This resulted in a significantly lower $\mathrm{E} / \mathrm{A}$ ratio in the diabetic twins than in their non-diabetic co-twins $(95 \% \mathrm{CI}-0.35$ to $-0.1, \mathrm{P}<0.001)$ or the controls $(95 \% \mathrm{CI}-0.79$ to -0.39 , $\mathbf{P}<0.001)$. Although the total mean velocity integrals (FVIA + FVIE) of diastolic filling were similar in twins and controls, the diabetic twins had significantly higher atrial filling fraction than either their co-twins $(95 \%$ CI 0.92 to $4.28 \%, \mathrm{P}<0.01$ ) or the controls ( 4.02 to $8.95 \%, P<0.001)$, indicating that a greater component of left ventricular diastolic filling was contributed by atrial systole. Furthermore, IVRT was significantly longer in the diabetic twins than in non-diabetic cotwins $(95 \%$ CI 5.41 to $11.88 \mathrm{~ms}, \mathrm{P}<0.001)$ and controls ( $95 \% \mathrm{CI}$ to 7.29 to $18.78 \mathrm{~ms}, \mathrm{P}<$ 0.001 ) (table 4), indicating a consistent difference in left ventricular diastolic function in the diabetic twins. The peak A velocity, A velocity integral, and atrial filling fraction were higher in the non-diabetic twins than controls, whereas the E/A ratio was lower in the non-diabetic twins than in the controls (table 4). To determine whether the differences in Doppler indices were simply the result of differences in heart rate, we selected twin pairs with heart rates within 5 beats $/ \mathrm{min}$ of each other (table 5). After matching 15 pairs for heart rate, the statistically significant differences in mean peak A velocity, E/A ratio, atrial filling fraction (all $P<0.03$ ), and IVRT $(P<0.005)$ between the diabetic and nondiabetic co-twins remained.

DIABETIC COMPLICATIONS

Retinopathy and persistent microalbuminuria 
Table 5 Diastolic variables after matching for heart rate $(n=15)($ mean $(S D))$

\begin{tabular}{lll}
\hline Variable & $\begin{array}{l}\text { Diabetic } \\
\text { twins }\end{array}$ & $\begin{array}{l}\text { Non-diabetic } \\
\text { twins }\end{array}$ \\
\hline Heart rate (beats/min) & $79(14)$ & $80(10)$ \\
E velocity (cm/s) & $68(15)$ & $67(9)$ \\
A velocity (cm/s) & $44(12)^{\star}$ & $36(5)$ \\
E/A ratio & $1 \cdot 57(0 \cdot 34)^{\star \star}$ & $1 \cdot 89(0 \cdot 41)$ \\
FVIE & $77(19)$ & $80(13)$ \\
FVIA & $30(10)$ & $25(5)$ \\
A filling \% & $28(6)^{\star}$ & $24(5)$ \\
IVRT (ms) & $71(10)^{\star \star}$ & $60(10)$
\end{tabular}

See footnote to table 4 for abbreviations. Statistical significant difference between twins ${ }^{*} P<0.03$ and ${ }^{\star} \mathrm{P}<0.005$.

Table 6 Correlations of echocardiographic variables between diabetic and their non-diabetic co-twins

\begin{tabular}{ll}
\hline Variables & Correlation coefficient $(r)$ \\
\hline LVEDDi & 0.72 \\
LVESDi & 0.65 \\
PwTi & 0.57 \\
IVSTi & 0.70 \\
E velocity & 0.48 \\
A velocity & 0.43 \\
A filling \% & 0.58 \\
IVRT & 0.67
\end{tabular}

See footnotes to tables 3 and 4 for abbreviations. All correlations were significant at $P<0.005$.

were not found in the non-diabetic twins but occurred in $38 \%$ and $23 \%$ of the diabetic twins respectively. In the diabetic twins, $17 / 160$ autonomic function tests $(11 \%)$ were abnormal compared with only $1 / 160(<0 \cdot 1 \%)$ in the non-diabetic twins (all $\mathrm{P}<0.001)\left(\chi^{2}\right.$ test).

\section{RELATION BETWEEN DATA}

Although there were differences in the echocardiographic measurements of left ventricular diastolic filling between the diabetic and non-diabetic twins, there were strong correlations between the diabetic and non-diabetic co-twins for each of these measurements (table 6). However, no correlation was demonstrated between the diabetic twins and controls or between the non-diabetic twins and controls, suggesting that the correlations between identical twins are caused by the influence of genetic factors (figure). To examine the impact of the duration of diabetes on left ventricular diastolic filling, the diabetic twins were divided into two groups according to their mean disease duration (14 years). Twins with diabetes for more than 14 years $(\mathrm{n}=20)$ had significantly greater peak $\mathrm{A}$ velocity (50 (11) v 39 (7) $\mathrm{cm} / \mathrm{s} ; \mathrm{P}<0.001)$

\section{Correlation of left} ventricular end diastolic dimension between diabetic and non-diabetic twins.

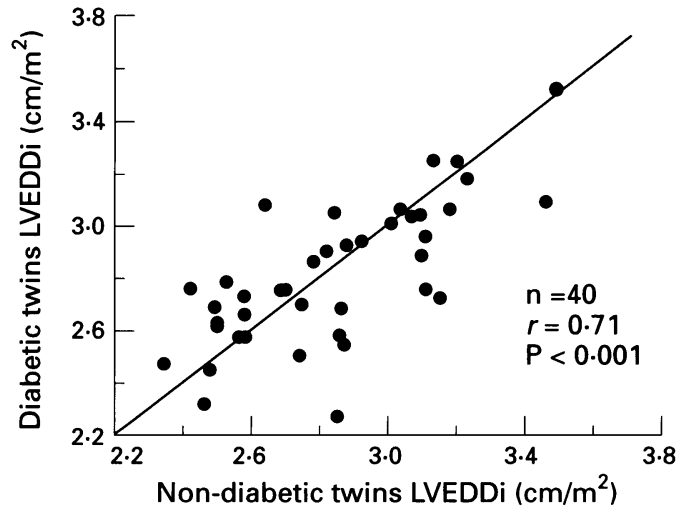

and A velocity integral (64 (17) v 50 (10); P < $0.001)$, greater atrial filling fraction $(35(5) v$ $31(5) \% ; \mathrm{P}<0.001)$ and lower $\mathrm{E} / \mathrm{A}$ ratio $(1.45(0.51) v 1.75(0.44) ; \mathrm{P}=0.02)$ than their non-diabetic co-twins. These differences were not detected in twins who had had diabetes for less than 14 years. In contrast, blood pressure and IVRT remained significantly different in the diabetic and their non-diabetic twins regardless of disease duration. Stepwise regression did not demonstrate an important influence of glycaemic control or blood pressure on any of the echocardiographic measurements of left ventricular diastolic function. A significant relation was detected between the diabetic complication index and IVRT $(P=0.01)$ : this significant relation was mainly related to the autonomic function score $(P=0.03)$. Further analysis showed that IVRT was significantly related to heart rate variation to deep breathing $(r=0.43$, $P=0.01)$ and heart rate response to standing $(r=0.35, \mathrm{P}=0.04)$, but not to the Valsalva manoeuvre $(\mathrm{P}=0.06)$.

\section{Discussion}

The study of monozygotic twins discordant for a specific disease entity provides a powerful means by which the influence of the disease on a particular organ function can be differentiated from the influence of genetic factors. For this reason, we studied the impact of type 1 diabetes on cardiac size and function in monozygotic twins who were discordant for diabetes. Genetic and also environmental influences, independent of sex, blood pressure, and age, have been shown to be important determinants of cardiac size and mass. ${ }^{14-16}$ Shared genetic factors may account for more than $60 \%$ of the total variance in left ventricular mass. ${ }^{16}$ In our study, we found strong correlations between the diabetic and non-diabetic twins for left ventricular end diastolic $(r=0.72)$ and end systolic $(r=0.65)$ dimensions, and interventricular septal thickness $(r=0 \cdot 70)$. However, there were no significant correlations between either the diabetic or the non-diabetic twins and the age and sex matched singleton control subjects in any of the echocardiographic measurements, reconfirming the important influence of both genetic and shared environmental factors upon the left ventricle. To identify the separate influence of genetic and environmental factors would need a different study design involving both identical and non-identical twins. Left ventricular end diastolic and end systolic cavity dimensions and fractional shortening were all similar in the diabetic twins, their co-twins, and the control subjects. Although all those studied were normotensive, the diabetic twins had higher blood pressures than their co-twins, but there were no differences in left ventricular wall thickness between the twin pairs.

Left ventricular diastolic function has been shown to be affected at an early stage in several myocardial diseases even when systolic function remains normal. ${ }^{17}{ }^{18}$ Several studies 
have used pulsed wave Doppler echocardiography to diagnose and characterise abnormal diastolic function by assessing the pattern of transmitral blood flow velocity during diastole. ${ }^{19-21}$ Left ventricular diastolic filling patterns by Doppler correlate closely with those obtained by contrast and radionuclide angiographic techniques. ${ }^{21} 22$ These velocity derived indices have proved to be sensitive indicators of impaired left ventricular filling in a wide range of myocardial diseases, ${ }^{20-22}$ even though they vary with age, heart rate, and left ventricular loading conditions. ${ }^{23}$ In our study, the diabetic twins had significantly greater peak $\mathrm{A}$ velocities and $A$ velocity integrals and reduced $\mathrm{E} / \mathrm{A}$ ratio compared with their non-diabetic co-twins. In addition, the contribution of left atrial contraction to left ventricular diastolic filling was also significantly greater in the diabetic twins. The difference in left ventricular diastolic filling between the diabetic and nondiabetic co-twins could not be explained by the faster heart rate in the diabetic twins because this difference persisted when 15 twin pairs with similar heart rates were compared. Importantly, these alterations in Doppler left ventricular filling patterns predominated in twins who had had diabetes for a long time, that is, more than 14 years.

Further independent evidence for altered diastolic function was provided by the prolonged isovolumic relaxation time in the diabetic twins, which is an acknowledged method of assessing diastolic function. We also demonstrated for the first time, that prolonged isovolumic relaxation time was related to cardiac autonomic function, particularly to the assessment of heart rate variability during deep breathing, which has been considered as one of the most sensitive test for the presence of cardiac autonomic neuropathy. ${ }^{12} 24$ The autonomic nervous system provides neurohumoral stimuli that modulate heart rate, and myocardial relaxation and contractility. In addition, it probably affects the loading conditions of the heart through the control of peripheral vascular resistance. Left ventricular systolic function and peak filling rate, a measure of diastolic function, have been demonstrated by radionuclide ventriculographic studies to be depressed in diabetic patients with autonomic neuropathy. ${ }^{25}$ In our study, diabetes duration did not influence isovolumic relaxation time, and previous studies have shown that diabetes duration did not influence the presence of autonomic neuropathy. ${ }^{26}$ The faster resting heart rate of the diabetic twins is most likely caused by subclinical autonomic neuropathy. ${ }^{24}$ However, the faster heart rate could not have resulted in the prolongation of isovolumic relaxation time because this difference persisted when 15 twin pairs with similar heart rates were compared. Furthermore, there was no correlation between the prolongation of isovolumic relaxation time and peak $A$ velocity, suggesting that they vary independently of each other. Thus if diastole can be divided into four phases (isovolumic relaxation, rapid filling, diastasis, and atrial contraction) then at least two of these, isovolumic relaxation and atrial contraction, are altered in the diabetic twins. There was no alteration in resting systolic function in our diabetic twins although systolic dysfunction may develop at a later stage or may be uncovered during exercise stress. ${ }^{927}$

These changes in left ventricular diastolic function in monozygotic twins with type 1 diabetes were unrelated to glycated haemoglobin concentrations measured at the time of the study, nor could they be explained by ischaemia, because according to previous studies coronary artery disease is uncommon in diabetic patients aged less than $40 .{ }^{589}$ Structural and metabolic abnormalities of diabetic myocardium have been reported and may have played a part in the functional changes we described. ${ }^{28} 29$ Increased deposition of collagen in the myocardium has been found in myocardial biopsy specimens ${ }^{928}$ obtained from patients with type 1 diabetes, and by in vivo studies of diabetic myocardium using an ultrasound backscatter technique. ${ }^{30}$

Our results show that both left ventricular dimension and function are under the influence of genetic and shared environmental factors, and that patients with type 1 diabetes have significant left ventricular diastolic dysfunction which may be related to disease duration and changes in autonomic function. The alterations in diastolic function manifested by dynamic changes in left ventricular filling from the early to late stages of diabetes are subtle and provide support for the existence of a specific diabetic heart muscle disease. Since genetic factors can exert important influences upon the left ventricle, subtle changes in cardiac function can best be detected by the study of monozygotic twins. Long-term serial follow up studies are necessary to determine whether these diabetic twins will progress to clinical heart failure, and Doppler echocardiography may offer a sensitive and non-invasive method to evaluate this progression.

We thank Mr G Davies, consultant ophthalmologist, King's College Hospital, Denmark Hill, London, for grading the retinal photographs, and Dr D Young, Senior Lecturer, Department of Statistics and Mathematics, Brunel University, Department of Statistics and Mathematics, Brunel University,
Middlesex, for statistical advice. This study was supported by Middlesex, for statistical advice. This study was supported by
the Sir Jules Thorn Charitable Trust, Diabetic Twins the Sir Jules Thorn Charitable Trust, Diabetic Twins
Research Trust and British Medical Association (Geoffrey Holt Award).

1 Garcia MJ, McNamara PM, Gordon T, Kanel WB. Morbidity and mortality in diabetics in the Framingham population. Diabetes 1974;23:105-11.

2 Kannel WB, Hjortland M, Castelli WP. Role of diabetes in congested heart failure: The Framingham study. $A m \mathcal{F}$ Cardiol 1974;34:29-34.

3 Shapiro LM, Leatherdale BA, Mackinnon J, Fletcher RF Left ventricular function in diabetes mellitus. II: Left ventricular function in diabetes mellitus. II: Relation between clinical features and
function. Br Heart $₹$ 1981;45:129-32.

4 Danielsen $R$, Nordrenhaug JE, Lien $E$, Vik-Mo $H$ Subclinical left ventricular abnormalities in young subjects with long-term type 1 diabetes mellitus detected by digitised M-mode echocardiography. Am $\mathcal{J}$ Cardiol 1987; digitised $M$

5 Bouchard A, Sanz N, Botvinick EH, Phillips N, Heilbron $\mathrm{D}, \mathrm{Byrd}$ III BF, et al. Non-invasive assessment of cardiomyopathy in normotensive diabetic patients between 20 and 50 years old. Am $\mathcal{F}$ Med 1989;87:160-6.

6 Sanderson JE, Brown DJ, Rivellese A, Kohner E. Diabetic Cardiomyopathy? An echocardiographic study of young diabetics. Br Med $\mathfrak{f}$ 1978;i:404-7. 
7 Zarich SW, Arbuckle BE, Cohen LR, Roberts M, Nesto RW. Diastolic abnormalities in young asymptomatic diabetic patients assessed by pulsed Doppler echocardiography. $\mathcal{F}$ Am Coll Cardiol 1988;12:114-120.

8 Krolewski AS, Koisinski EJ, Warram JH, Leland SO, Busick EJ, Asmal AC, et al. Magnitude and determinants of coronary artery disease in juvenile-onset insulindependent diabetes mellitus. Am f Cardiol 1987;59: $750-5$

9 Fisher BM, Gillen G, Lindop GBM, Dargie HJ, Frier BM. Cardiac function and coronary arteriography in asymptomatic Type 1 (insulin-dependent) diabetic patients: evidence for a specific diabetic heart disease. Diabetologia 1986;29:706-12.

10 National Diabetes Data Group. Classification and diagnosis of diabetes mellitus and other categories of glucose sis of diabetes mellitus and other categori
intolerance. Diabetes $1979 ; 28: 1039-57$.

11 Sarna S. Probabilities of concordance of twins with respect to genetic markers: A general formulation. Acta Genet to genetic markers: A general formulatio

12 Ewing DJ, Clarke BF. Diagnosis and management of diabetic autonomic neuropathy. $\mathrm{Br} \mathrm{Med} \mathcal{f} 1982 ; 285$ : 916-8.

13 Sahn DJ, DeMaria T, Kisslo J, Weymen A. The committee on M-mode standardization of the American Society of Echocardiography. Recommendations regarding quantitation in M-mode echocardiography: Results of a survey of echocardiographic measurements. Circulation 1978; 58:1072-82.

14 Adams TD, Yanowitz FG, Fisher AG, Ridges JD, Nelson $\mathrm{AG}$, Hagan $\mathrm{AD}$, et al. Heritability of cardiac size: an echocardiographic and electrocardiographic study of monozygotic and dizygotic twins. Circulation 1985;71: monozy.

15 Bielen E, Fagard R, Amery A. Inheritance of heart structure and physical exercise capacity: A study of left structure and physical exercise capacity: A study of left
ventricular structure and exercise capacity in 7-year-old ventricular structure and exercise

16 Verhaaren HA, Schieken RM, Mosteller M, Hewitt JK, Eaves LJ, Nance WE. Bivariate genetic analysis of left ventricular mass and weight in pubertal twins (The
Medical College of Virginia Twin Study). Am $\mathcal{f}$ Cardiol 1991;68:661-8.

17 Hirota Y. A clinical study of left ventricular relaxation. Circulation 1980;62:756-63.

18 Aroesty JM, McKay RG, Heller GV, Royal HD, Als AV, Grossman W. Simultaneous assessment of left ventricular systolic and diastolic dysfunction during pacing-induced ischaemia. Circulation 1985;71:889-900.

19 Appleton CP, Hatle LK, Popp RL. Relation of transmitral flow velocity pattern to left ventricular diastolic function: new insights from a combined haemodynamic and Doppler echocardiographic studies. $\mathcal{F} \mathrm{Am}$ Coll Cardiol 1988;12:426-40.

20 Shapiro LM, Gibson DG. Patterns of diastolic function in left ventricular hypertrophy, Br Heart f 1988;59:438-45.

21 Stoddard MF, Pearson AC, Kern MJ, Ratcliff J, Mrosek DG, Labovitz AJ. Left ventricular diastolic function: comparison of pulsed Doppler echocardiography and haemodynamic indexes in subjects with and without coronary artery disease. $f \mathrm{Am}$ Coll Cardiol 1989;13. 327-36.

22 Rokey R, Kuo LC, Zoghbi WA, Limacher MC, Quinones MM. Determination of left ventricular diastolic filling with pulsed Doppler echocardiography: comparison with with pulsed Doppler echocardiography: compa

23 Van Dam I, Fast T, De Boo T. Normal diastolic filling patterns of the left ventricle. Eur Heart $₹$ 1988;9:165-71.

24 Watkins PJ. Diabetic autonomic neuropathy. $N$ Engl $f$ Med 1990;322:1078-9.

25 Erbas T, Erbas B, Gedik O, Biberoglu S, Bekdik CF Scintigraphic evaluation of left ventricular function and correlation with autonomic cardiac neuropathy in diabetic patients. Cardiology 1992;81:14-24.

26 Donaghue KC, Bonney M, Simpson JM, Schwingshandl J, Fung ATW, Howard NJ, et al. Autonomic and peripheral nerve function in adolescents with and without diabetes. Diabetic Med 1993;10:664-71.

27 Vered Z, Battler A, Segal P, Liberman D, Yershalmi Y, Berezin $M$, et al. Exercise-induced left ventricular dysfunction in young men with asymptomatic diabetes melfunction in young men with asymptomatic diabetes mellitus (diabe

28 van Hoeven KH, Factor SM. A comparison of the pathological spectrum of hypertensive, diabetic, and hypertensive-diabetic heart disease Circulation 1990;82:848-55.

29 Rodrigues B, McNeill JH. The diabetic heart: metabolic causes for the development of a cardiomyopathy Cardiovasc Res 1992;26:913-22.

30 Perez JE, McGill JB, Santiago JV, Schechtman KB Waggoner AD, Miller JG, et al. Abnormal myocardia acoustic properties in diabetic patients and their correlation with the severity of disease. 7 Am Coll Cardiol 1992 19:1154-62. 